\title{
PROBABILISTIC APPROACH TO DOMAIN SELECTION FOR INTEGRATING THE NORMAL'S FIELD IN 3D RECONSTRUCTION
}

\author{
Bogdan Rusyn, Oleksiy Lutsyk, Yarema Varetskyy \\ Physico-Mechanical Institute of the NAS of Ukraine \\ 5, Naukova str. Lviv, 79060, Ukraine \\ Email: rusyn@ipm.lviv.ua, olutsyk@ipm.lviv.ua, j.varetskyy@ipm.lviv.ua
}

\begin{abstract}
The method of integrating normal's field is improved by using the proposed algorithm for breaking local areas based on Schwartz equations. It was proposed local criteria of splitting normal's onto local areas, which is crucial for the next step of building the sequence chain. The process of classification in case of reducing the training set to select regions of integration is investigated. The reason for the effect of retraining is conditioned with a minimal number of errors on the training sample. It is shown that stratification of algorithms by mistakes and increasing their similarities reduce the likelihood of the retraining. Proposed approaches are realized in software and are suitable for the broad class of real surfaces. Copyright (C) Research Institute for Intelligent Computer Systems, 2015. All rights reserved.
\end{abstract}

Keywords: 3D reconstruction, photometric stereo, normal integration, classification, normals field.

\section{INTRODUCTION}

Three-dimensional reconstruction is used to obtain the shape of a real object utilizing one of the methods of reconstruction. The main method of reconstruction, which is the most widely used, is stereo reconstruction. However, in this reconstruction arise such difficult problems as image matching and camera calibration, which leads to significant computational complexity. Given this all together, makes use of photometric reconstruction methods [1, 2, 3, 4]. These methods allow obtaining the surface orientation based on information of the several images with different illumination directions, but fixed cameras. Photometric reconstruction methods are effective for a certain class of the surfaces that have Lambertian reflection of the light, illuminated by the ideal light source and are smooth without sharp transitions.

An essential step in photometric reconstruction is normal field surfaces integration of the reconstructed surface. There are several approaches developed to solve this problem: direct integration [5], the integration field normals by calculating deviations [6], using the methods of analysis in the frequency domain [7]. In terms of computational complexity, the superior characteristics have direct integration methods while as optimization approaches provide high quality reconstruction. The disadvantages of optimization approaches include computational complexity and disadvantages of choosing regularization. In cases of non-integration of the surface methods based on calculating deviations show better property.

Given the shortcomings of the proposed approach is the need to develop a method of determining the shape of the surface normals of the field. It is proposed to develop a direct integration of local areas where local areas are selected based on statistical criteria.

\section{STATE OF THE ARTS}

Today many different techniques have been developed for $3 \mathrm{~d}$ reconstruction. Laser scanning [12], structured light systems [13, 14] and multiview stereo methods directly reconstruct an oriented point cloud, from which a closed surface can be derived by applying one of the surface fitting methods. Also there are approaches that only rely on information about the surface normals. All of those approaches require surface normals integration.

Currently exists many approaches of surface normals integration. The base method developed by Horn and Brooks based on the calculation of variations. The drawbacks are it requires initial information integration and extremely computationally cost. Frankot proposed frequency domain method with batter properties, but still with artifacts. Galliani and Breuss proposed robust surface normal integration by Eikonal equation [9], which is based on the method of Ho. This method 
is a fast and highly robust, but it suitable for smooth surfaces.

We proposed the approach which is based on the direct integration scheme of $\mathrm{Wu}$ and $\mathrm{Li}$ [5]. It shows better results for real image surfaces in addition to convex space- time multi-view approach proposed by Oswald and Cremers [10] and Kameras [11]. By break the normals field onto local areas of interest according to Schwartz equations the integration process become more robust in local areas of interest. Also we proposed probabilistic approach to the problem of region selection to build the sequence chain of local areas. The effect of using this approach significantly prevail random approaches.

\section{INTEGRATION OF NORMALS}

In the step of integration of normals field appeared problems of non-integration regions as normals not satisfy the Schwartz equation. To solve this problem was proposed modified method of integrating the surface normals based on the partition of the field normals to the local areas that satisfy a certain approximation of Schwartz equation. According to Schwartz equation it was proposed local criteria of splitting normals onto local areas (1).

$$
\sum_{i}\left|\frac{\partial p_{i}}{\partial y_{i}}-\frac{\partial q_{i}}{\partial x_{i}}\right| \leq e
$$

where $p=\frac{\partial z}{\partial x}, q=\frac{\partial z}{\partial y}-$ partial derivatives, $e-\mathrm{a}$ fixed predetermined value.

After splitting the field of normals onto local areas, normals integrating are implemented by single areas, where previous region, already integrated set to an initial approximation to the next, bordering it. The integration is carried out according to the expression (2)

$$
z(I)=\sum_{i=1}^{P}\left(\int_{M_{i}}(p d x+q d y)\right)
$$

where $P-$ the number of regions of all normal field.

The integration areas are under scheme (Fig. 1), where each successive local area can generate several different ways of integration. As a result of the integration it is proposed to average the different ways of integration. This allows us to estimate mean and deviation from the mean in any point of the surface.
We are facing to task, which one area of integration is the best in terms of optimality. We are trying to find the answer by applying probabilistic approach and building the sequence chain of local area integration.

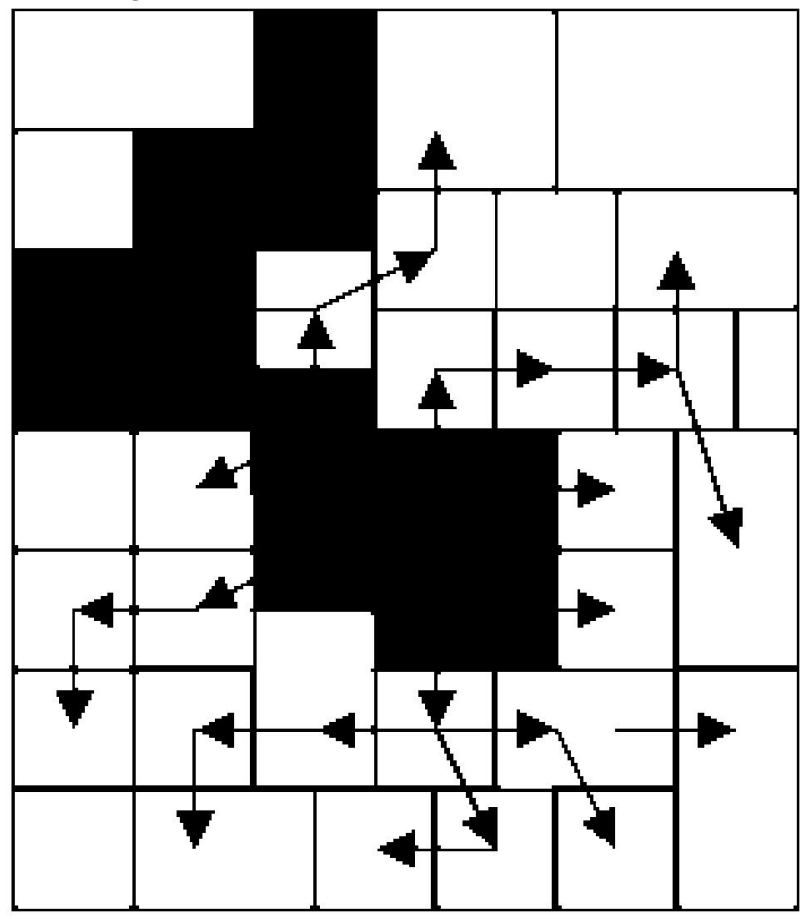

Fig. 1 - Integrating scheme of related areas of normal field.

\section{DETERMINE THE SEQUENCE CHAIN OF LOCAL AREA}

An important problem that arises in the task of integration normals field by local area is building the sequence chain of local area. This is achieved by estimation of the training set. The training set is built directly from the normals of local area. The problems occur when the training set is limited or require shrinking, depending on classifier.

The quality of classifications that are based on rank vote and using the separating hyperplane, characterized by the concept of margin, which is the distance of the object from the parting hyper plane. The greater the margin the better is classifier. However, if all objects from the training set or most of them are about the same margin and grouped next to each other, in this case content of their information decrease. This means that instead of all objects, we can leave one or more used for training the classifier [8].

The reason for the effect of retraining is conditioned due to the fact that the algorithm with a minimal number of errors on the training sample [9]. Retraining will be greater, the greater the composition of the algorithms is used. This is true for algorithms taken from the distribution randomly and independently. In the case when algorithms are 
dependent, (in a real situation they usually are dependent) assumed that retraining decrease. The effect of the retraining can occur even when choosing only one of the two algorithms. Stratification of algorithms by mistakes and increasing their similarities reduce the likelihood of the retraining.

For example, consider doublet sampling algorithm. Each algorithm covers a number of objects of the training set. If to use the internal criteria (for example, in the case of metric classifiers), we can estimate the stability of the coating and narrow the number of covered objects by a given level of stability. Thus, in order to cover more objects it is necessary to apply greater number of algorithms. These algorithms must be similar and, at the same time, have a different error rates. This is the best strategy for building modern composition algorithms. However, when using test data, where composition algorithms are not adapted, the classification error can be quite different from the minimum, obtained in the training data.

On the other hand, seems to be an interesting task to determine the amount of redundant information in the training data. The feasibility of reducing the training data is important, because for each case of classification decreases the number of objects and other classes that are harmful for classification. It is necessary to estimate the average class size, providing the required level of error frequency. Reducing the size of training data also means reducing class size in the testing stage.

Consider the highlights of probabilisticcombinatorial approach for metric classifiers, which allows estimating the impact of effect of class size shrinking on the probability of correct classification, desired local area to build the sequence chain of local area. First, we present combinatorial approach.

Present the results of classification $A$ as a binary sequence ordered by a minimum distance of objects the size $t A=\{0,1\}$ of the confidence interval $I_{t}$, where 1 is associated with own pattern, and $0-$ stranger pattern.

An example of such sequences is presented in Fig. 2.

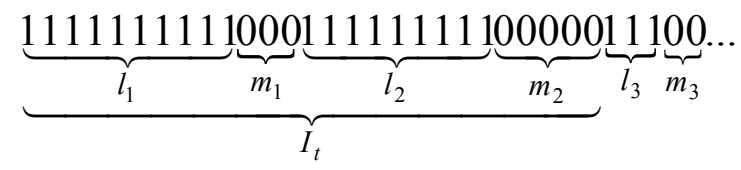

Fig. 2 - Results of classification in the form of binary sequences.

Evaluation of the effect of reducing the size of the training data makes it possible to define a data structure.
It is important that among the $\mathrm{k}$ nearest neighbours are relative or absolute majority of the own class patterns among stranger patterns. Consider a simple case that involves a relative majority of own class patterns. The successful classification requires for $\mathrm{k}$ nearest neighbours the condition (3).

$$
\left|\bigcup_{i} \widetilde{l}_{i}\right|>\bigcup_{i} \tilde{m}_{i} \mid, i=1,2,3 \ldots,
$$

where $\tilde{l}_{i}, \tilde{m}_{i}$ - groups formed after the shrink of information covering classes.

Groups are homogeneous sequence elements. A sequence includes all classes pattern. Although in general correspondence between the number of groups and the number of classes does not exist.

Condition of correct operation of the classifier: $\operatorname{ENT}\left(\frac{k}{2}\right)+1 \leq s^{*}$. Determine the probability that a sequence of patterns of the length of their class will be selected in combinatorial method of $s^{*}$ patterns. These probabilities are characterize the coverage of uncompressed class sequence of $\left|\bigcup_{i} l_{i}\right|$ patterns, including selected $s^{*}$. In addition, we find also the likelihood that some patterns of strange classes would not be selected in relevant way.

Denote the probability of misclassification caused by the strange class patterns for groups as $m_{i}$ and $q_{j}$.

$$
\begin{aligned}
& q_{1}=P\left(\inf \left(\bigcup_{i} m_{i} \mid\right) \geq E N T\left(\frac{k}{2}\right)+1\right) ; \\
& q_{2}=P\left(\inf \left(\bigcup_{i} m_{i} \mid\right)+\left|m_{i+1}\right| \geq E N T\left(\frac{k}{2}\right)+1\right) ; \\
& q_{3}=P\left(\inf \left(\bigcup_{i} m_{i} \mid\right)+\left|m_{i+1}\right|+\left|m_{i+2}\right| \geq E N T\left(\frac{k}{2}\right)+1\right) ; \ldots \\
& q_{j}=P\left(\inf \left(\bigcup_{i} m_{i} \mid\right)+\bigcup_{j} m_{i+j-1} \mid \geq E N T\left(\frac{k}{2}\right)+1\right) ; \ldots
\end{aligned}
$$

The probability of misclassification caused by strange class patterns:

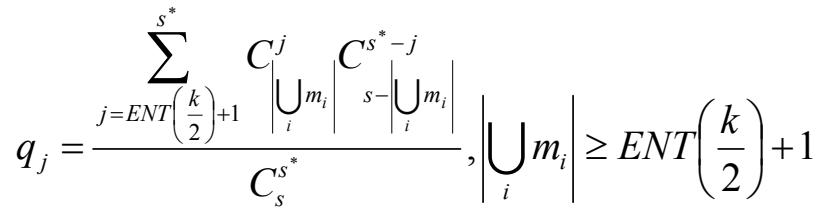


And the probability for an arbitrary sequence of own class patterns (4).

$$
P_{q_{i}}=\frac{\sum_{j=E N T\left(\frac{k}{2}\right)+1}^{s^{*}} C_{\left|\bigcup_{i}^{j} l_{i}\right|} C^{s^{*}-j}-\bigcup_{i} l_{i} \mid}{C_{s}^{s^{*}}} .
$$

The probability of successful classification using the classifier determined by the product of probability $P_{q_{i}}$ and probability addition $q_{j}$ :

$$
\begin{aligned}
& P_{j}=P_{q_{j}}\left(1-q_{j}\right)=\frac{\sum_{j=E N T\left(\frac{k}{2}\right)+1}^{s^{*}} C_{\mid}^{j} \bigcup_{i} l_{i} C^{s^{*}-j} s-\bigcup_{i} l_{i} \mid}{C_{s}^{s^{*}}}-
\end{aligned}
$$

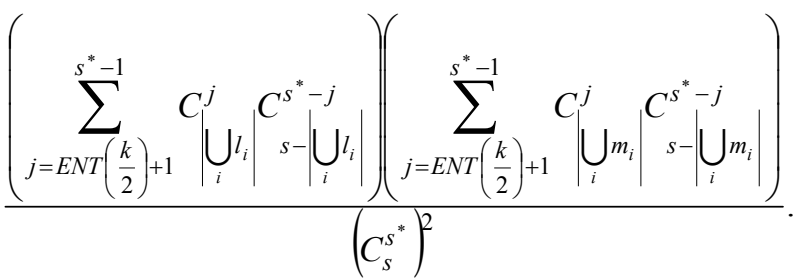

The idea of the probabilistic approach is next. Unlike combinatorial approach, where classification results known exactly, now defined probability of the existence of an original sequence.

Since the probability of the existence of arbitrary sequence is rather small, especially for large training samples, than probability determined by the existence of homogenous sequences of patterns. This probability is determined by the last object in the sequence, like probability of substitution of the object by other database objects.

\section{TESTING}

All of the proposed algorithms for the integration of normals and determine the sequence chain of local area where utilized in processing of test and real data.

In Fig. 3 presented the main stages of reconstruction including an analysis of a series of images, the definition of normals field and integration by proposed method.

The Research of the methods in implemented software was carried out on a wide class of test and real images of surfaces.

Fig. 4 shows a result of the reconstruction of the standard test images database for photometric stereo. In Fig. 5 presented results of reconstruction of real surface of the material.

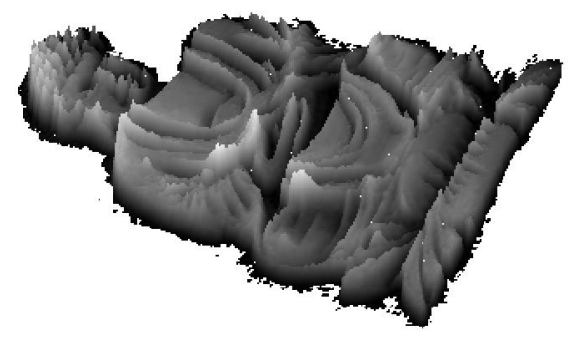

Fig. 3 - Surface difference along paths of integration.

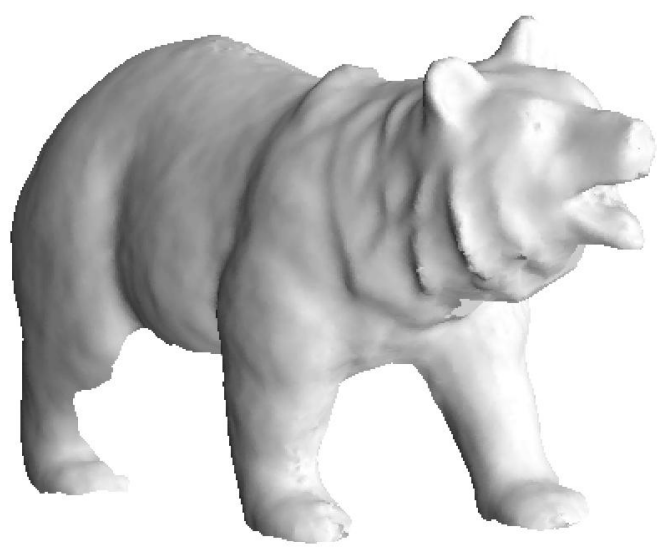

Fig. 4 - The results of evaluating the depth coordinate values for test image.

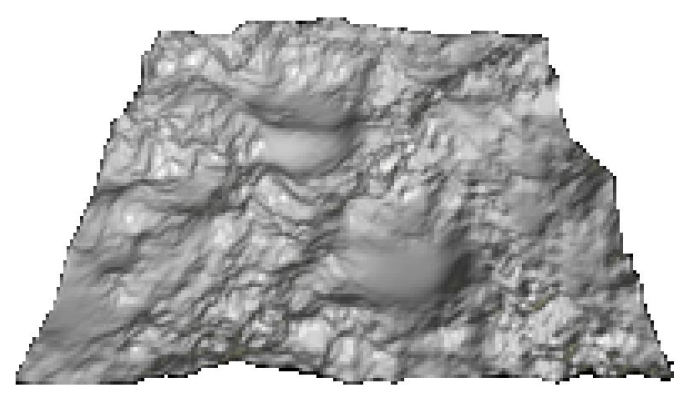

Fig. 5 - The result of evaluating the depth coordinates for real surface material.

According to the results (Fig. 6), proposed approach exceeds the existing methods in $5-10$ percent depending on test surface.

\begin{tabular}{|l|c|c|}
\hline Method & $\begin{array}{c}\text { Surface } \\
\text { Bottle }\end{array}$ & $\begin{array}{c}\text { Surface } \\
\text { Monum }\end{array}$ \\
\cline { 2 - 3 } & nmse & nmse \\
\hline Woodham & 0,1142 & 0,1023 \\
\hline Tsai & 0,1057 & 0,0971 \\
\hline Horn & 0,0921 & 0,0868 \\
\hline Zeng & 0,0972 & 0,0922 \\
\hline Proposed approach & 0,0904 & 0,0876 \\
\hline
\end{tabular}

Fig. 6 - Comparison of different methods no quality of evaluation the depth coordinates. 


\section{CONCLUSIONS}

The problem of normals field Integration is a well known and important in the aria of 3D reconstruction. During working was developed a method, able to obtain the shape from the normals field based on direct integration approach (Fig. 6).

We proposed to break the normals field onto local areas of interest according to Schwartz equations. It was proposed local criteria of splitting normals onto local areas, which is crucial for the next step of building the sequence chain. Also, proposed probabilistic approach to the problem of region selection to build the sequence chain of local areas. It is investigated the process of classification in case of reducing the training set (arias of normals field). Proposed approach of normals field Integration is suitable for the broad class of real surfaces. Finally, implementation of the proposed approach allowed obtaining the gain in 10 percent (Fig. 6).

\section{REFERENCES}

[1] R. J. Woodham, Determining surface curvature with photometric stereo, in Proceedings of the IEEE Conference on Robotics and Automation, Scottsdale, USA, 1989, pp. 36-42.

[2] L. B. Wolf, Surface curvature and contour from photometric stereo, in Proceedings of the Defence Advanced Research Project Agency Image Understanding Workshop (DARPA), Los Altos, USA, 1987, pp. 821-824.

[3] H. D. Tagare, R. J. P. de Figueredo, A theory of photometric stereo for a class of diffuse nonLambertian surfaces, IEEE Transactions on Pattern Analysis and Machine Intelligence, (13) 2 (1991), pp. 133-152.

[4] M. S. Drew, Shape and specularity from color, Technical Report CSS/LCCR TR-93-01, Simon Fraser University, Burnaby, 1993.

[5] Z. Wu, L. Li, A line-integration based method for depth recovery from surface normals, in Proceedings of the $9^{\text {th }}$ IEEE Conference on Pattern Recognition, Rome, Italy, 1988, pp. 591-595.

[6] J. Ho, J. Lim, M. H. Yang, D. J. Kriegman, Integrating surface normal vector susing fast marching method, in Proceedings of the European Conference on Computer Vision (ECCV), Graz, Austria, May 7-13, 2006, pp. 239-250.

[7] J. D. Durou, J. F. Aujol, and F. Courteille, Integrating the normal field of a surface in the presence of discontinuities, in Proceedings of the Energy Minimization Methods in Computer Vision and Pattern Recognition (EMMCVPR), Lecture Notes in Computer Science, (5681) 2009, pp. 261-273.

[8] B. P. Rusyn, V. A. Tayanov, O. A. Lutsyk, Upper-bound estimates for classifiers based on a dissimilarity function, Cybernetics and system analysis, Eds. Springer-Verlag, (48) 4 (2012), pp. 592-600.

[9] S. Galliani, M. Breuß, Y. C. Ju, Fast and robust surface normal integration by a discrete Eikonal equation, R. Bowden, J. Collomosse, K. Mikolajczyk (Eds.), in Proceedings of the $23^{\text {rd }}$ British Machine Vision Conference (BMVC), Surrey, UK, September 3-7, 2012, BMVA Press, pp. 1-11.

[10] M. Oswald, D. Cremers. Surface normal integration for convex space-time multi-view reconstruction, in Proceedings of the British Machine Vision Conference (BMVC), Nottingham, UK, September 1-5, 2014, pp. 61-71.

[11] D. Cremers, K. Kolev, Multiview stereo and silhouette consistency via convex functionals over convex domains, IEEE Transactions on Pattern Analysis and Machine Intelligence, (33) 6 (2011), pp. 1161-1174.

[12] P. Shenga, P. Bomark, O. Broman and O. Hagman, 3D phase-shift laser scanning of log shape, BioResources, (9) 4 (2014), pp. 7593-7605.

[13] S. Zhang and S. T. Yau, High-resolution, realtime $3 \mathrm{D}$ absolute coordinate measurement based on a phase-shifting method, Optics Express, (14) 7 (2006), pp. 2644-2649.

[14] P. Payeur, D. Desjardins, Structured light stereoscopic imaging with dynamic pseudorandom patterns, Image Analysis and Recognition, Lecture Notes in Computer Science, Springer, (5627) (2009), pp. 687-696.

[15] S. Ferrari, I. Frosio, V. Piuri, N. A. Borghese, Automatic multiscale meshing through HRBF networks, IEEE Transactions on Instrumentation and Measurement, (54) 4 (2005), pp. 1463-1470.

[16] S. Ferrari, F. Bellocchio, V. Piuri, N. A. Borghese, A hierarchical RBF online learning algorithm for real-time 3-D scanner, IEEE Transactions on Neural Networks, (21) 2 (2010), pp. 275-285. 


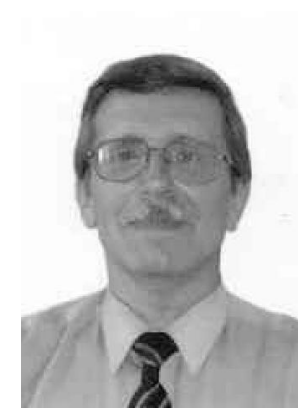

Prof. Dr. Bogdan Rusyn, Head of Department "Methods and systems for processing, analysis and identification of images" Physico-Mechanical Institute of the NAS of Ukraine. $\mathrm{He}$ is the author of 300 scientific papers, 10 books, 20 patents. Related research areas are image processing, pattern recognition, $3 D$ reconstruction, methods of data compression, biometric identification and digital signal processing.

Dr. Oleksiy Lutsyk Researcher of Department "Methods and systems for processing, analysis and identification of images" Physico-Mechanical Institute of the NAS of Ukraine. He is the author of 36 scientific papers. Related research areas are image processing, pattern recogintion $3 D$ reconstruction and neural networks.

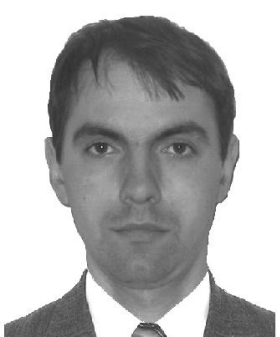

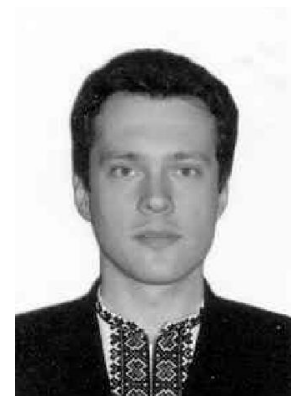

Dr. Yarema Varetskyy Researcher of Department "Methods and systems for processing, analysis and identification of images" Physico-Mechanical Institute of the NAS of Ukraine. He is the author of 25 scientific papers, 1 books, 1 patents. Related research areas are image processing, $3 D$ reconstruction, biometric identification and computational geometry. 